\title{
A novel method for producing 3D models of paranasal sinuses for forensic anthropology applications
}

Madeline Robles $\underline{\text { Ruth M. Morgan }}$ (D) \&arolyn Rando

Received 25 Mar 2020, Accepted 28 Apr 2020, Published online: 24 May 2020

To cite this article:

Madeline Robles, Ruth M. Morgan \& Carolyn Rando (2020) A novel method for producing 3D models of paranasal sinuses for forensic anthropology applications, Australian Journal of Forensic Sciences, DOI: $\underline{10.1080 / 00450618.2020 .1766113}$

\begin{abstract}
Human identifications are made difficult when the remains are severely fragmented or burned. In cases such as these, alternative methods of identification become vital. Three-dimensional reconstructions have increased the potential for utilizing qualitative and quantitative analysis of anatomical structures within forensic anthropology approaches. This paper presents a method to produce three-dimensional reconstructions of paranasal sinuses for biological analysis of skeletal remains. Previous published research has quantified the variability of the paranasal sinuses and has begun to explore their ability to provide biological information within forensic science contexts. However, the complex anatomical structure of the sinuses has led to significant limitations in the ability to produce three-dimensional reconstructions for analysis using an automatic approach. Therefore, this new method for developing three-dimensional models of the paranasal sinuses using an automatic approach that is suitable for a large sample size is timely. It offers a new pathway to more sophisticated methods of analysis that ultimately offer the potential to provide valid and robust distinctions between individuals and identifications in crime reconstructions.
\end{abstract}

KEYWORDS: Forensic science, forensic anthropology, segmentation, paranasal sinuses, three-dimensional modelling

\section{Introduction}


The paranasal sinuses consist of four distinct sinuses located in the skull (including maxillary sinuses, ethmoid, sphenoid and frontal sinus). Researchers have quantified the variability of the paranasal sinuses between individuals and have begun to explore their utility in assessing biological patterns $1-3$. The structure of the paranasal sinuses has been shown to have great potential for discriminating between individuals, particularly when considering the frontal and sphenoidal sinus are perhaps the most variable cavity in the human body $\mathbf{2}^{-}$ $z$ (see Figure 1 and Figure 2). Previously, these structures have been analysed using a range of methods including codifying and comparing certain features $4-6$ or by manually procuring 2D measurements or Cartesian coordinates from x-ray or

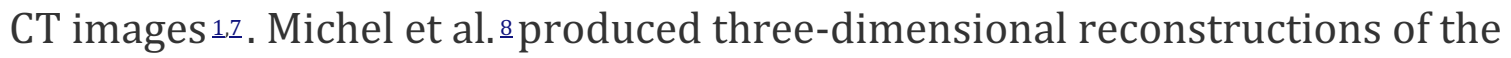
frontal sinus from sinus CT scans and found the total volume of the sinus to be the most discriminatory datum for sex determination. It was found that 3D reconstructions of anatomical structures similar to the frontal sinus provided more reliable data than 2D measurements given that the sinus and other

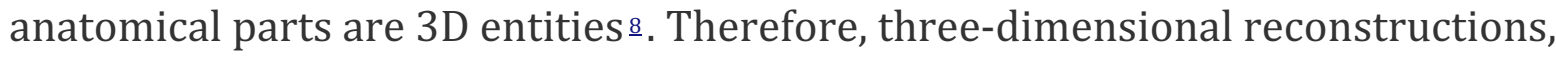
which are integral to virtual anthropology, are increasingly being shown to enhance the potential for qualitative and quantitative morphological analysis . However, the complex and variable anatomy of the paranasal sinuses has made it difficult to produce three-dimensional models that enable analysis to be undertaken in a standardized and time-efficient manner $9-12$. As a result, there is currently no standard approach for producing models of the paranasal sinuses. Moreover, of the few published methods $9-14$ available for producing threedimensional reconstructions of the paranasal sinuses, none of them employ an automatic approach that is suitable for a large sample size within forensic anthropology contexts.

Figure 1. Three-dimensional model of the paranasal sinuses produced by CT scans from University College London Hospital.
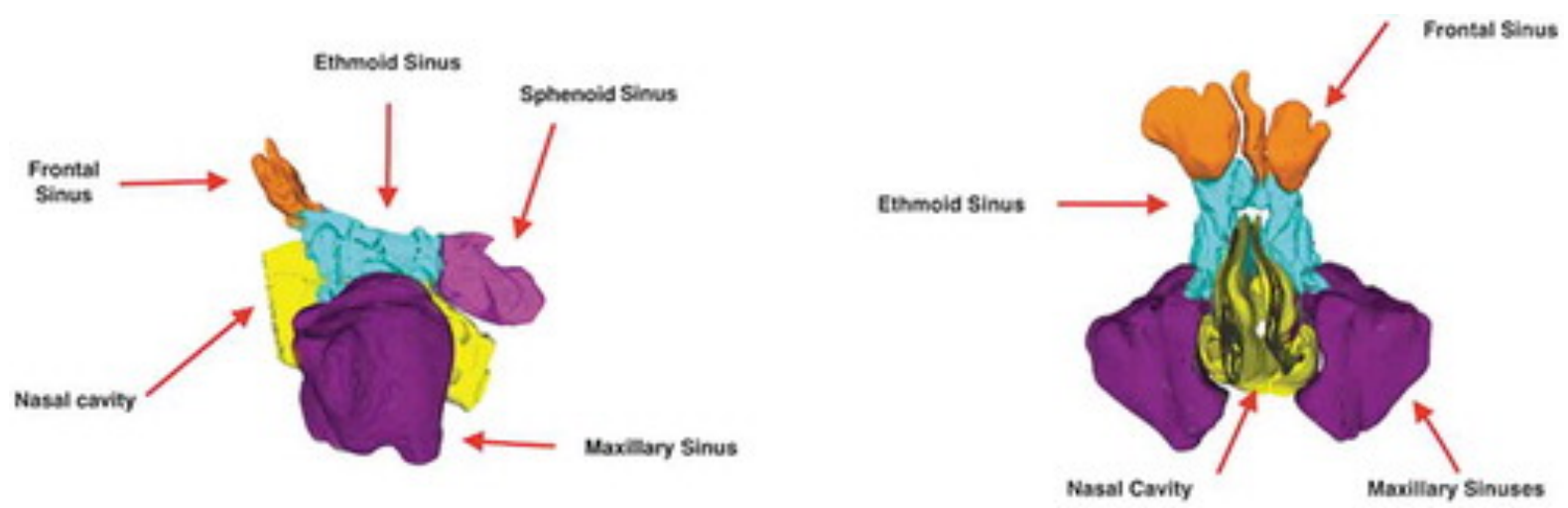

Display full size

Figure 2. The location of the paranasal sinuses in the skull. 

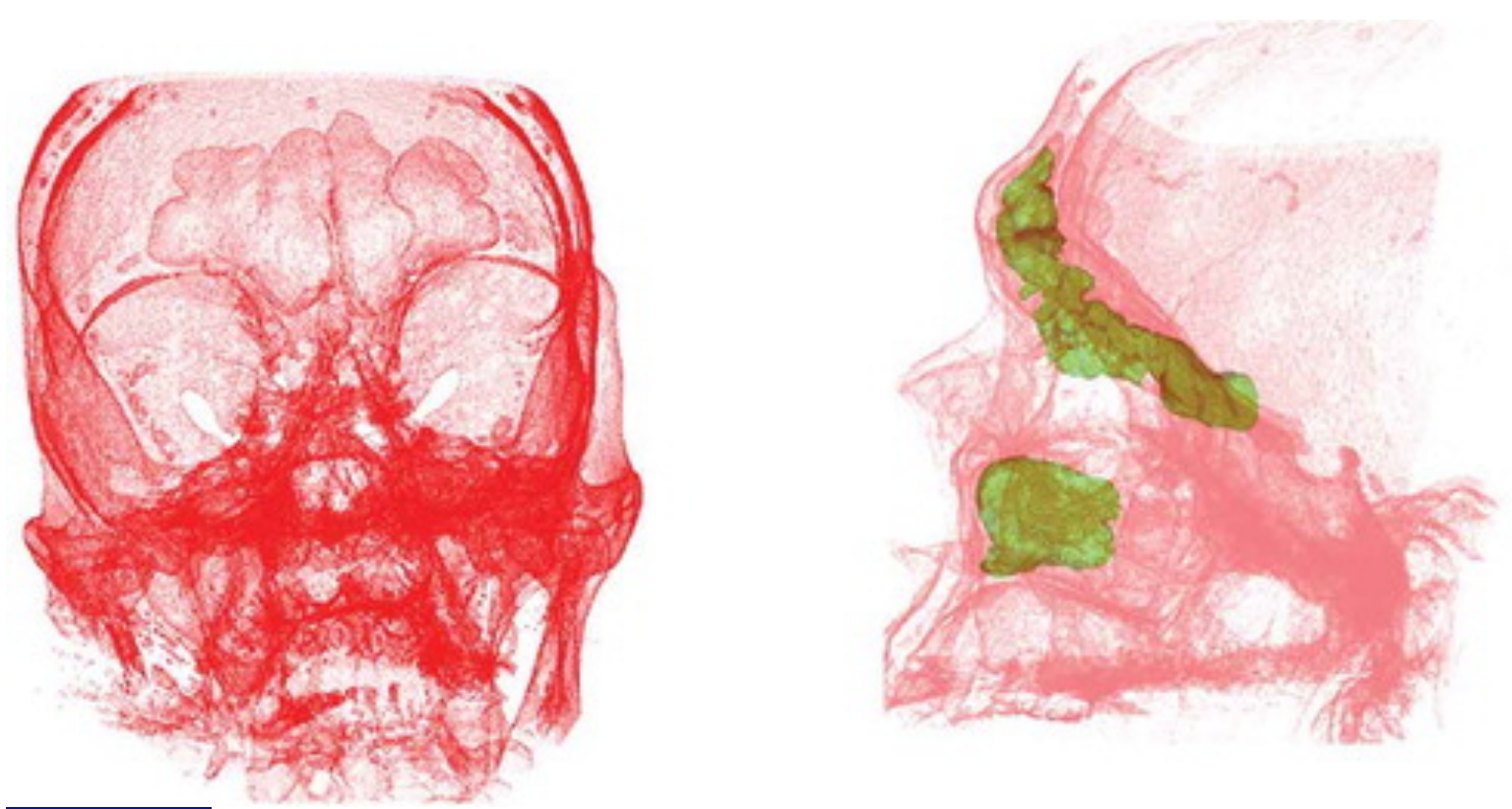

Display full size

The complex anatomical structure of the paranasal sinuses makes the development of standardized methods of segmentation and three-dimensional reconstruction limited. Previous studies $3.9-14$ have therefore needed to apply both automatic and manual methods to create 3D models. However, manual methods pose two distinct issues relating to time and expertise. The first issue is the significant amount of time 9 manually segmenting larger sample sizes takes, and the second issue is that this approach relies on more instances of human decision-making that significantly rely on additional expertise 9 . There is therefore considerable potential in developing an automatic approach that is time efficient and less reliant on the specialized training of the observer for broader applications within crime reconstructions. This paper presents a method to produce 3D models of the paranasal sinuses that allows for further measurement and analysis for potential use in forensic anthropology applications.

The method presented here offers an approach to:

- Enable three-dimensional reconstruction of the varying and complicated structures of the paranasal sinuses at a larger scale.

- Limit the need for specialized training.

- Allow for easy and transparent decision making throughout the modelling process.

The guiding principle in the development of this method was to create an empirical approach in order to ensure that 3D reconstructions could be produced in a reliable and reproducible manner. In so doing, this method provides a tool that can produce empirical measures that have the potential to contribute to an evidence base that underpins and enables transparency of the analyses of the 
data made by an examiner, and the inferences made that lead to final conclusions in crime reconstructions.

\section{Materials and methods}

The production of anatomical three-dimensional models requires radiographic scans. In this case, 500 anonymized computed tomography (CT) scans of the paranasal sinuses were provided by the picture archiving and communications (PACS) department from University College London Hospital (UCLH) in DICOM (Digital Imaging and Communications in Medicine) imaging format. The DICOM format allows a visualization platform to view the scans and ultimately produce the three-dimensional models. The program3D Slicerтм (Version 4.9.0) was selected as the most promising form of visualization as it contains a DICOM Viewer, allows for easy export and import of STL (stereolithography) files to other platforms, and most importantly, it is free to access and open-source 15. This ease of access facilitates practical application in forensic science where financial resources are often limited, and its use in examining paranasal sinuses in previously published studies further confirms its utility in this study 16 . Additionally, 3D Slicerтм allows for the possibility of the three-dimensional models to be electronically distributed globally for additional virtual analysis should an investigation require them 17 . Research 18 has also shown no significant difference between models produced from commercial and free software. Furthermore, the precision and accuracy of 3D models produced from CT scans is well documented 19-21. This capability contrasts with other professional viewing platforms which due to high costs and insufficient accessibility are not practical for research or use in a forensic science context.

\section{D model production}

\section{Uploading data}

In order to produce the three-dimensional models of the paranasal sinuses, CT scans were uploaded into 3D Slicer ${ }^{\top M}$. The CT scan of the individual automatically opens within the DICOM viewer. The clinical details of a particular CT scan are included in the 'metadata section' of 3D Slicer ${ }^{T M}$. The information given in this section include the scanner used and the slice thickness of the CT scan.

\section{Crop volume}

The complex and variable anatomy of the paranasal sinuses provides challenges in producing three-dimensional models automatically because the nasal cavity merges with the paranasal sinuses. The key to producing three-dimensional reconstructions of the paranasal sinuses without the inclusion of the nasal cavity 
is by cropping the volume of the CT scan. Cropping the volume of the CT scans to only include the Region of Interest (ROI) is an important step in the overall production of the paranasal sinus reconstructions. This allows for faster computer processing $\underline{22}$ and harnesses the anatomical complexity of the paranasal sinuses to produce a modelling process that is faster overall 22 (see Figure 3 ).

Figure 3. This process to be repeated for each sinus.

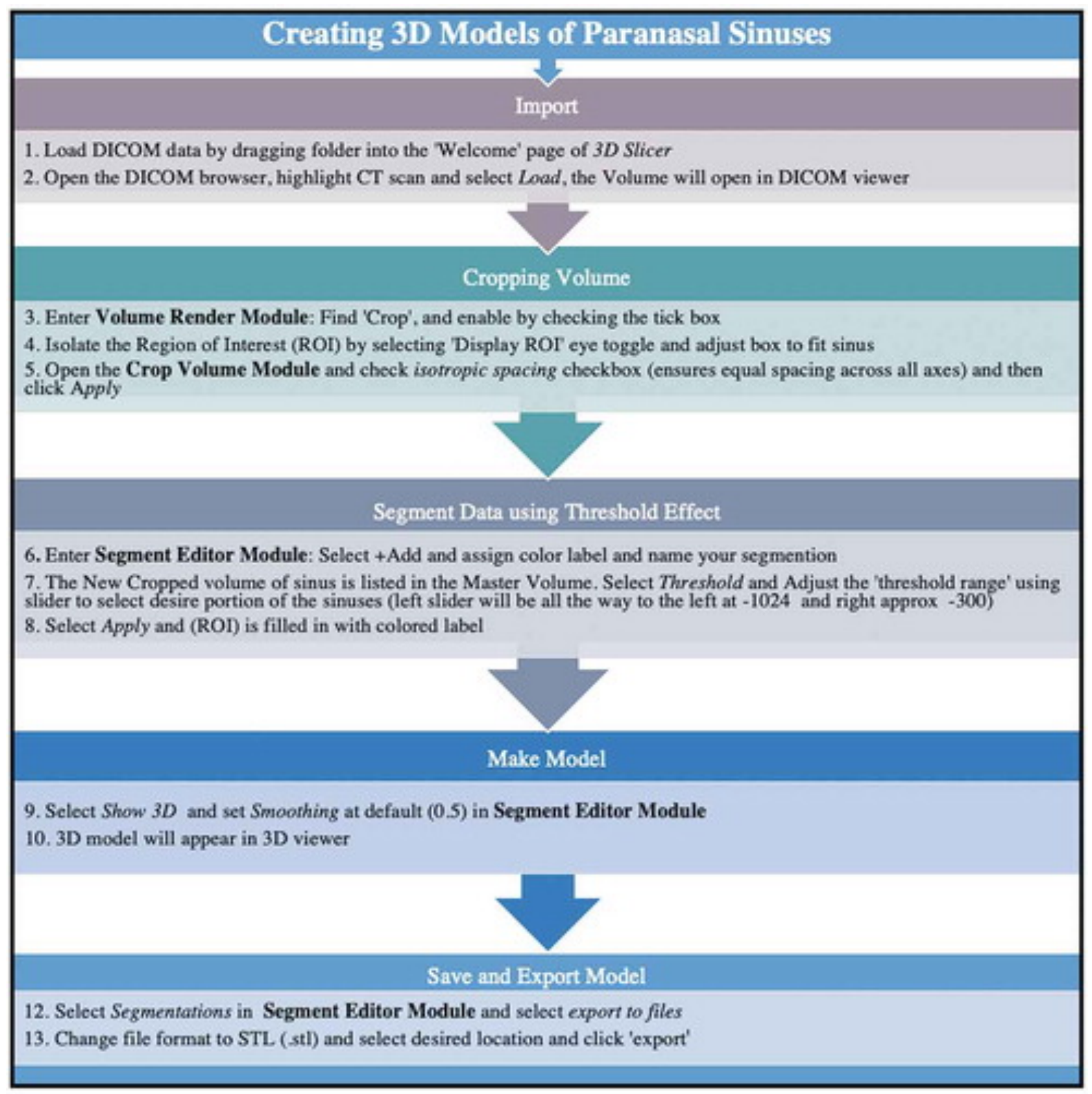

\section{Display full size}

This method does not manually segment the CT scans because this would greatly slow down the modelling process and is not realistic for a large database. A manual approach would also require specialized knowledge of the structure of each paranasal sinus, which limits its utility in future forensic science settings. Therefore, the most practical and time efficient method for creating the collective paranasal models is to apply the 'Crop Volume' module to the CT scan to only 
include one sinus at a time, and automatically segment each single sinus individually. The process was repeated for each sinus in a single CT dataset. Cropping the CT scans uniformly eliminates irrelevant portions of the CT scan to leave only the regions of interest needed for the three-dimensional reconstruction. This includes uniformly eliminating the nasal cavity, leaving the entire dimensions of the individual sinus needed for segmenting. Cropping the CT scans also produced an automatic approach, which ultimately allowed for faster segmentation process.

Figures 4 and $\underline{5}$ presents the cropping process applied to a CT scan of the frontal sinus. In the case of the frontal sinus, the upper and lateral limits of this structure were often easily defined. However, the extensive nature of the nasal cavity made the lower boundary of the frontal sinus less evident in some instances. To ensure uniformity in the segmentation of the frontal sinus, this method adopted a widely accepted approach $12 \underline{23}$ that established the 'baseline' of the frontal sinus as tangential to the upper margin of the orbits. This was adjusted for this study to be the location where the volume of the CT scan was cropped (see Figure 5). This approach was easily employed and replicated for each 3D reconstruction of the frontal sinus.

The process of cropping the volume of the CT scans provided a simple and uncomplicated solution to the segmenting and thresholding process by eliminating the need to manually add or remove unintentionally segmented areas. This approach also reduced the active decision-making throughout the segmenting process that may have influenced the construction of the model and ultimately the final interpretations and conclusions drawn.

\section{Segmentation}

The segmentation process is integral in developing three-dimensional models. Therefore, it was important that this procedure was consistent and accurate in its representation of the paranasal sinuses. There are two approaches to segmentation within $3 D$ Slicer ${ }^{T M}$ : manual and automatic. The manual approach requires the user to examine each slice individually within a CT scan to highlight the appropriate portions of the CT scan 10 . The number of slices within a single CT scan requires manually observing hundreds of slices. It is not a viable option for a larger database particularly if there are significant time-constraints 210 .

Therefore, this method employed automatic segmentation by applying the 'global thresholding' effect on the cropped CT scans wherein the segmentation automatically applied to all of the slices within the CT dataset sinus at once. Applying global thresholding to the cropped CT scan for each sinus is a novel approach in producing three-dimensional models of the paranasal sinuses.

To begin segmenting, a 'threshold range' was established. The 'segment editor' module and the 'threshold effect' module was selected to provide the range (see Figure 4). The average threshold range to depict bone and tissue densities 
falls between 500-3000 Hounsfield Units (HU) in 3D Slicerтm 22 (see Figure 4). However, as the sinuses are air-filled the threshold range was reflected to select pixels that were closest to true black. This typically ranged from $(-1024$ to -300 HU) (see Figure 5).

Figure 4. Threshold range that captures the skull.
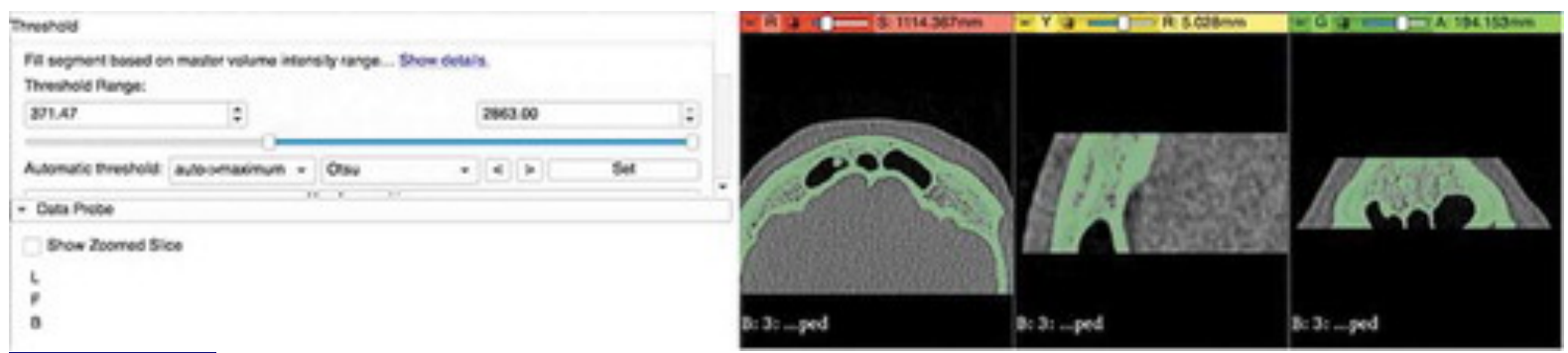

\section{Display full size}

Figure 5. Threshold range applied in this study that captured the paranasal sinuses.
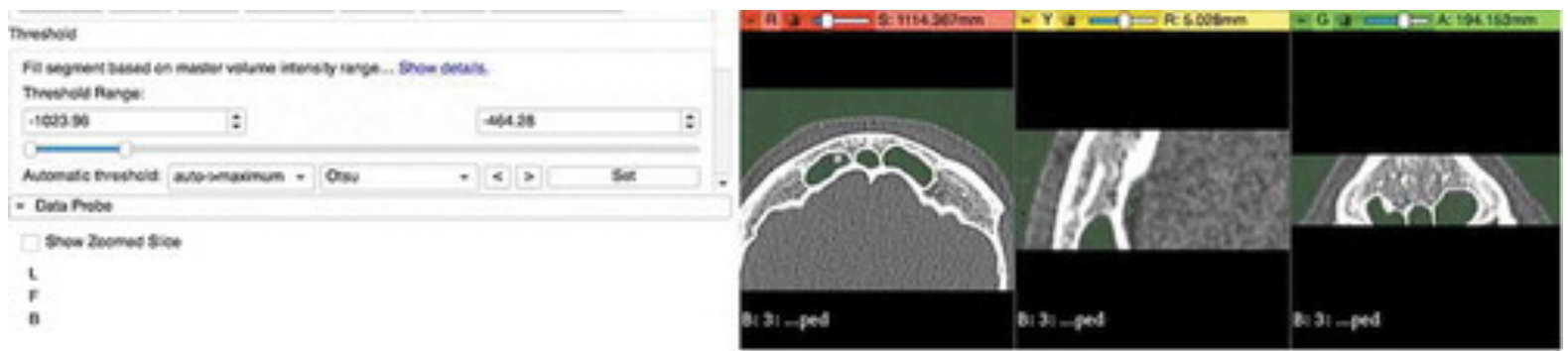

\section{Display full size}

\section{Final model}

Once the appropriate portions of the CT scans were selected for thresholding, the models were smoothed to $.5(0=$ no smoothing and $1=$ max smoothing $)$ default followed by utilizing the 'Show3D Model' function (see Figure 6). There is no observable or metric difference between auto-smoothed and nonauto-smoothed models 20 . Indeed, employing 'global thresholding' for the segmentation process can be used in a general sense to make a 3D model of any object or specimen. However, the steps involved with cropping are specific to this structure as ' $g l o b a l$ thresholding' would not be able to produce a model of the paranasal sinuses that excludes the nasal cavity unless the CT dataset was cropped (see Figure 1). The process of cropping and segmenting as outlined in this paper must be repeated for each sinus within a CT scan. The cropping process allows for each sinus to be segmented at once with the caveat that each sinus has to be segmented separately.

Figure 6. Image of the excess faces and vertices included in the three-dimensional models in MeshLab ${ }^{\text {тM }} \underline{24}$. 


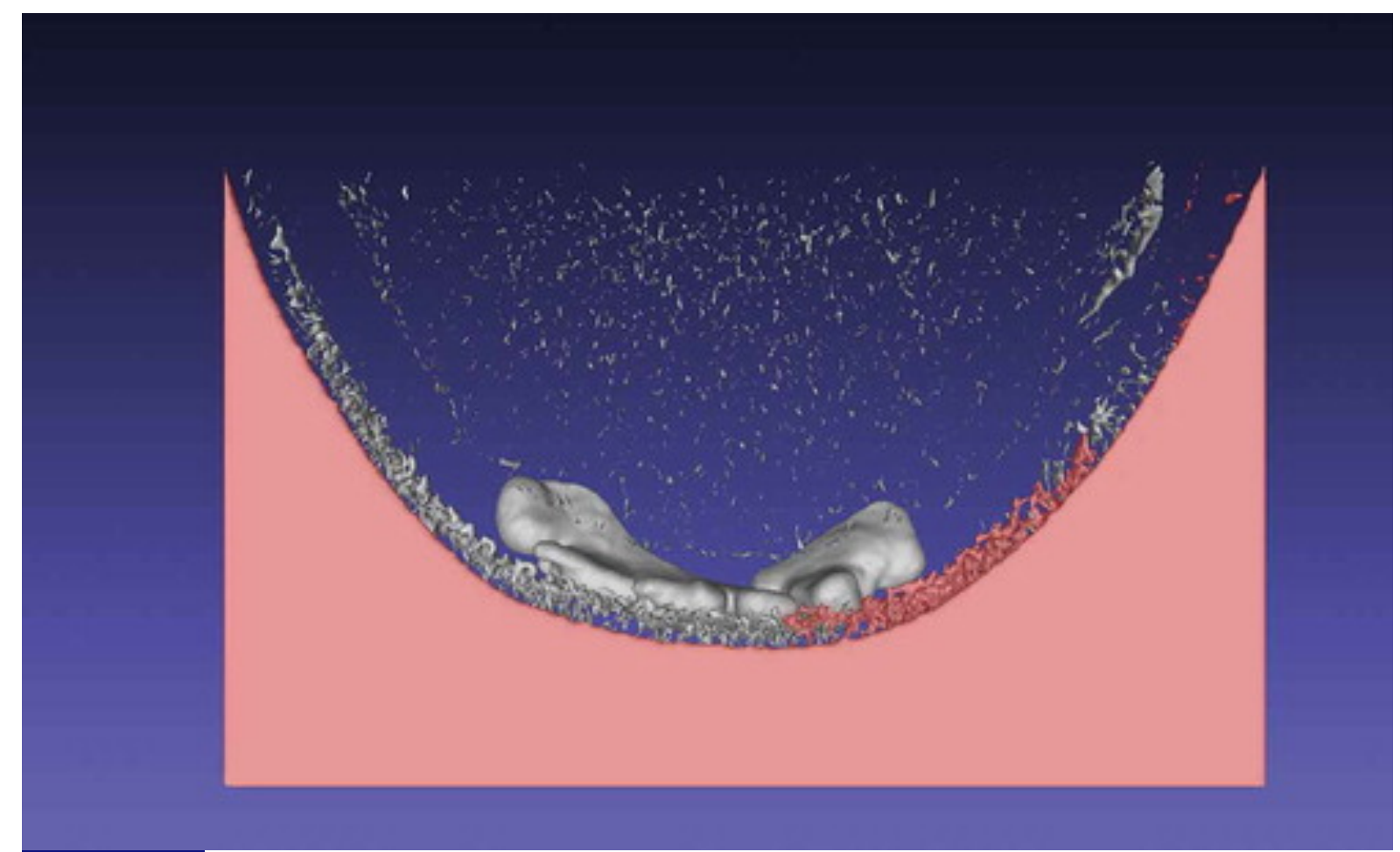

Display full size

The standard threshold range for capturing the sinuses uses pixels closest to true black and may result in background noise (see Figure 6). Therefore, the excess faces and vertices included in the final model must be removed. This can be achieved in post-processing by saving the final STL file and transferring it to any visualization platform where the excess segmentations may be eliminated if needed. Figure 7 provides a view of the final three-dimensional models of each sinus ready for analysis. The application of this approach took approximately 3045 minutes per CT scan with a user familiar with 3D Slicerт. This is in contrast to a manual approach that can take an expert nearly 10 hrs per CT scan 210 . This method produced three-dimensional models of the paranasal sinuses with speed and consistency that allows further research into the potential of employing these structures for human identification.

Figure 7. Final image after the removal of excess faces and vertices of the threedimensional models. 


\begin{tabular}{|c|c|c|}
\hline $\begin{array}{c}\text { Frontal } \\
\text { Sinus }\end{array}$ & \\
\hline $\begin{array}{c}\text { Ethmoid } \\
\text { Sinus }\end{array}$ & \\
\hline $\begin{array}{c}\text { Sphenoid } \\
\text { Sinus }\end{array}$ & \\
\hline $\begin{array}{c}\text { Maxillary } \\
\text { Sinuses }\end{array}$ & \\
\hline
\end{tabular}

\section{Display full size}

\section{Conclusion}

The variable anatomy of the paranasal sinuses between individuals hinders the ability to produce a uniform method in producing three-dimensional models at a faster rate using a larger database. The complexity of this structure has forced previous studies to employ manual approaches which are not only time consuming but also are significantly dependent upon the specialized experience and/or expertise of the observer. The method presented here addresses these issues by employing an automatic approach and enables the production of consistent models using open and free software that allows for additional analysis to become of potential use in forensic science applications. This method therefore offers a tool that is easy to use, and thereby enables practical applications in forensic science scenarios that must usually accommodate time and resource constraints. The context of forensic anthropology and its role in reconstructing crime events is an important consideration that should be incorporated into new tools and approaches if they are to be implementable in real world crime reconstructions.

\section{Acknowledgments}


The authors would like to thank Dr Sherry Nakhaeizadeh for her final edits and contributions to this paper. The authors would also like to thank the picture archiving and communications department at University College London Hospital for their help in procuring the database of CT scans that made this study possible following approval from the Health Research Authority and the NHS Research Ethics Committee.

\section{Disclosure statement}

No potential conflict of interest was reported by the author(s).

\section{References}

1. ChristensenA. Assessing the variation in individual frontal sinus outlines. Am J Phys Anthropol. 2005;127(3):291-295. doi:10.1002/ajpa.20116 [Crossref], [PubMed], [Web of Science $\AA$ ], [Google Scholar]

2. AuffretM, GaretierM, DialloI, AhoS, Ben SalemD . Contribution of the computed tomography of the anatomical aspects of the sphenoid sinuses to forensic identification. J Neuroradiol. 2016;43(6):404-414. doi:10.1016/j.neurad.2016.03.007 [Crossref], [PubMed], [Web of Science ${ }^{\circledR}$ ], [Google Scholar]

3. CohenO, WarmanM, FriedM, Shoffel-HavakukH, AdiM, HalperinD, LahavY. Volumetric analysis of the maxillary, sphenoid and frontal sinuses: a comparative computerized tomography based study. Auris Nasus Larynx. 2018;45(1):96-102. doi:10.1016/j.anl.2017.03.003 [Crossref], [PubMed], [Web of Science $®$ ], [Google Scholar]

4. CulbertW, LawF . Identification by comparison of roentgenograms. J Am Med Assoc. 1927;88(21):1634. doi:10.1001/jama.1927.02680470020009 [Crossref], [Google Scholar]

5. YoshinoM, MiyasakaS, SatoH, SetaS . Classification system of frontal sinus patterns by radiography. Its application to identification of unknown skeletal remains. Forensic Sci Int. 1987;34(4):289-299. doi:10.1016/0379-0738(87)900417 [Crossref], [PubMed], [Web of Science $®$ ], [Google Scholar]

6. KirkN, WoodR, GoldsteinM . Skeletal identification using the frontal sinus region: a retrospective study of 39 cases. J Forensic Sci. 2002;47(2):15250J. doi:10.1520/JFS15250J [Crossref], [Web of Science ®], [Google Scholar]

7. CoxM, MalcolmM, FairgrieveS . A new digital method for the objective comparison of frontal sinuses for identification. J Forensic Sci. 2009;54(4):761-772. doi:10.1111/j.1556-4029.2009.01075.x [Crossref], [PubMed], [Web of Science ß], [Google Scholar]

8. MichelJ, PaganelliA, VaroquauxA, PiercecchiMartiM, AdalianP, LeonettiG, DessiP . Determination of sex: interest of frontal sinus 3D reconstructions. J Forensic Sci. 2015;60(2):269-273. doi:10.1111/15564029.12630 [Crossref], [PubMed], [Web of Science ®], [Google Scholar] 
9. TingelhoffK, EichhornK, WagnerI, KunkelM, MoralA, RilkM, WahlF, BootzF . Analysis of manual segmentation in paranasal CT images. Eur Arch

Otorhinolaryngol. 2008;265(9):1061-1070. doi:10.1007/s00405-008-0594-

$\mathrm{z}$ [Crossref], [PubMed], [Web of Science ${ }^{\circledR}$ ], [Google Scholar]

10. PirnerS, TingelhoffK, WagnerI, WestphalR, RilkM, WahlF, BootzF, EichhornK . CT-based manual segmentation and evaluation of paranasal sinuses. Eur Arch Otorhinolaryngol. 2009;266(4):507-518. doi:10.1007/s00405-008-0777-

7 [Crossref], [PubMed], [Web of Science $\left.{ }^{\circledR}\right]$ [Google Scholar]

11. BuiN, OngS, FoongK. Automatic segmentation of the nasal cavity and paranasal sinuses from cone-beam CT images. Int J Comput Assist Radiol Surg. 2014;10(8):1269-1277. doi:10.1007/s11548-014-1134-5 [Crossref], [PubMed], [Web of Science $₫]$ [Google Scholar]

12. SinhaA, LeonardS, ReiterA, IshiiM, TaylorR, HagerG . Automatic segmentation and statistical shape modeling of the paranasal sinuses to estimate natural variations. Medl Imaging. 2016;9784:97840. [Google Scholar]

13. KapakinS . The paranasal sinuses: three-dimensional reconstruction, photo-realistic imaging, and virtual endoscopy. Folia Morphol. 2016;75(3):326-333.

doi:10.5603/FM.a2016.0006 [Crossref], [PubMed], [Web of Science ®], [Google Scholar]

14. SalahZ, BartzD, DammannF, SchwadererE, MaassenM, StraßerW . A fast and accurate approach for the segmentation of the paranasal sinus. In: Meinzer H-P, Handels $\mathrm{H}$, Horsch A, Tolxdoff T, editors. Bildverarbeitung für die Medizin 2005. Berlin,Heidelberg: Springer; 2005. p. 93-97. [Crossref], [Google Scholar]

15. FedorovA, BeichelR, Kalpathy-CramerJ, FinetJ, Fillion-

RobinJ, PujolS, BauerC, JenningsD, FennessyF, SonkaM, et al. 3D Slicer as an image computing platform for the Quantitative Imaging Network. Magn Reson Imaging. 2012;30(9):1323-1341.

doi:10.1016/j.mri.2012.05.001 [Crossref], [PubMed], [Web of Science ®], [Google Scholar]

16. GachP, Tuchtan-TorrentsL, DelteilC, AdalianP, PiercecchiM, EbertL, GorincourG . Virtual reconstruction of paranasal sinuses from CT data: A feasibility study for forensic application. Diagn Interv Imaging. 2019;100(3):163-168.

doi:10.1016/j.diii.2018.11.011 [Crossref], [PubMed], [Web of Science ®], [Google Scholar]

17. FranklinD, SwiftL, FlavelA . 'Virtual anthropology' and radiographic imaging in the Forensic Medical Sciences. Egypt J Forensic Sci. 2016;6(2):31-43. doi:10.1016/j.ejfs.2016.05.011 [Crossref], [Google Scholar]

18. AbdullahJ, AbdullahA, HadiH, HuseinA, RajionZ . Comparison of STL skull models produced using open-source software versus commercial software. Rapid Prototyp J. 2019;25(10):1585-1591. doi:10.1108/RPJ-08-2018-0206 [Crossref], [Web of Science ß], [Google Scholar]

19. ColmanK, DobbeJ, StullK, RuijterJ, OostraR, van RijnR, van der MerweA, de BoerH, StreekstraG . The geometrical precision of virtual bone models derived from clinical computed tomography data for forensic anthropology. Int J Legal Med. 2017;131(4):1155-1163. doi:10.1007/s00414-017-1548-

$\mathrm{z}$ [Crossref], [PubMed], [Web of Science $(\mathbb{Q}$ ], [Google Scholar]

20. CarewR, MorganR, RandoC . A preliminary investigation into the accuracy of 3D modeling and 3D printing in forensic anthropology evidence reconstruction. J Forensic 
Sci. 2018;64(2):342-352. doi:10.1111/1556-4029.13917 [Crossref], [PubMed], [Web of Science $\AA$ ], [Google Scholar]

21. DereliA, ZeybekV, SagtasE, SenolH, OzgulH, AcarK. Sex determination with morphological characteristics of the skull by using 3D modeling techniques in computerized tomography. Forensic Sci Med Pathol. 2018;14(4):450-459.

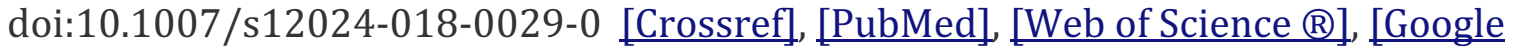
Scholar]

22. LiY, LuisE . 3D printing of medical models from CT-MRI images: A practical step-by-step guide. Singapore: Partridge Publishing; 2016. [Google Scholar]

23. LibersaC, FaberM . Étude anatomo-radiologique du sinus frontal chez l'enfant. Lille Med. 1958;3:453. [Google Scholar]

24.CignoniP, CallieriM, CorsiniM, DellepianeM, GanovelliF RanzugliaG. MeshLab: an OpenSource Mesh Processing Tool. In: ScaranoV, De ChiaraR, ErraU , editors. Sixth Eurographics Italian Chapter Conference. Salerno, Italy: The Eurographics Association; 2008. p. 129-136. [Google Scholar] 